\title{
Expression of chemokine CCL20 in ulcerative colitis
}

\author{
HAIFENG ZHANG ${ }^{1}$, WANE ZHONG ${ }^{2}$, GUOXIONG ZHOU ${ }^{1}$, \\ XIAOLING DING ${ }^{1}$ and HAIQING CHEN ${ }^{1}$ \\ ${ }^{1}$ Department of Gastroenterology, Affiliated Hospital of Nantong University, Nantong 226001; \\ ${ }^{2}$ Department of Gastroenterology, The Sixth Hospital of Ningbo, Zhejiang 315040, P.R. China
}

Received May 7, 2012; Accepted September 4, 2012

DOI: $10.3892 / \mathrm{mmr} .2012 .1088$

\begin{abstract}
The aim of the current study was to investigate the expression of chemokine CCL20 in ulcerative colitis (UC) patients and in dextran sulfate sodium (DSS)-induced experimental colitis in mice. The expression of the CCL20 protein in colonic mucosa was detected in $65 \mathrm{UC}$ patients and in 30 normal patients. A total of 40 female BALB/c mice were divided into a control and model group; the latter was fed a 5\% DSS solution ad libitum for 7 days to induce experimental colitis. The disease activity index (DAI) and histological score were assessed and the expression of CCL20 mRNA and protein was determined. CCL20 was expressed in the UC group. It was either weakly expressed or not expressed in the normal control group. Additionally, the expression of CCL20 in the UC group was significantly higher compared with that in the control groups $(\mathrm{P}<0.01)$. The expression levels of CCL20 mRNA and protein were significantly increased in the model group compared with those in the control group $(\mathrm{P}<0.01)$ and positively correlated with the severity of colonic inflammation. The results from the present study demonsrtate that CCL20 positively correlates with UC. CCL20 may play a significant role in local damage and pathological changes in UC and may serve as a potential target for therapy.
\end{abstract}

\section{Introduction}

The exact pathogenesis of ulcerative colitis (UC) remains unknown. However, a number of factors, including the histopathology of colonic lesions and the beneficial effects of

Correspondence to: Dr Guoxiong Zhou, Department of Gastroenterology, Affiliated Hospital of Nantong University, Nantong 226001, 20 Xisi Road, P.R. China

E-mail: zhouguoxiong@medmail.com.cn

Abbreviations: UC, ulcerative colitis; DSS, dextran sulfate sodium; DAI, disease activity index; H\&E, hematoxylin and eosin; RT-PCR, reverse transcription-polymerase chain reaction

Key words: ulcerative colitis, chemokine, CCL20, pathological changes corticosteroid therapy, point toward immunological involvement. Ulcerated lesions in UC are accompanied by a prominent infiltration of inflammatory cells, including $\mathrm{T}$ lymphocytes, macrophages, neutrophils and plasma cells. In immunological pathogenesis, cytokines are considered to play critical roles. Such factors are secreted by immune and non-immune cells. These small polypeptides have extensive biological functions, including regulating cell-to-cell signaling, adjusting the immune response and regulating inflammation. These small proteins may be divided into 3 classes: promoters of inflammation and anti-inflammatory and growth factors. In terms of UC pathogenesis, the balance between pro- and anti-inflammatory factors is considered to be particularly significant.

Chemotactic factors (chemokines) belong to a class of inflammatory molecules that plays a significant role in promoting the development of UC. Chemokines may be divided into 4 groups: CXC, CC, C and CX3C. CCL20 belongs to the $\mathrm{CC}$ group of chemotactic agents. It is strongly chemotactic for lymphocytes and recruits lymphocytes and dendritic cells (DDs) into epithelial tissue. Immature DCs (imDCs) are selectively attracted by CCL20. Therefore, CCL20 plays a role in the formation of mucosal lymphoid tissue. A number of research efforts have been aimed at inhibiting chemokines as a means of treating UC (1-7).

In the current study, dextran sulfate sodium (DSS) was used to induce UC in mice $(1,2,8,9)$. Immunohistochemistry, RT-PCR and western blotting were used to detect CCL20 in this experimental mouse model of colitis. Immunohistochemistry was also used to detect the CCL20 levels in 65 patients with $\mathrm{UC}$ and in 30 normal controls. Overall, this study examines the role of CCL20 in UC.

\section{Patients and methods}

Patients. Colonic biopsies were obtained from 65 consenting patients with UC (34 females and 31 males; median age, 44 years; range, 16-75 years) undergoing colonoscopy for diagnostic purposes as approved by the Institutional Review Board of the Affiliated Hospital of Nantong University. The diagnoses were based on clinical and endoscopic parameters. Endoscopic appearance of the colonic mucosa was assessed according to the criteria of Murano et al $(10)$ : mild $(n=22)$, moderate $(n=26)$ and severe $(n=17)$. Histological disease activity was assessed according to the criteria of Truelove and Richard: mild $(n=23)$, moderate $(n=24)$ and severe $(n=18)$. Additional colonic biopsies 
were obtained as the controls from consenting normal patients $(n=30 ; 8$ females and 22 males; median age, 42.5 years; range, 20-65 years) undergoing endoscopy to rule out neoplastic disease by pathological examination.

Grouping of experimental animals and establishment of the model. A total of 40 female adult BALB/c mice (mass, 14-20 g) were purchased from the Experimental Animal Center, Medical School of Nantong University (Nantong, China). The animals were kept in cages with controlled temperature $\left(23 \pm 2^{\circ} \mathrm{C}\right)$ on a 12 -h light-dark cycle and were randomly divided into 2 groups ( $\mathrm{n}=20$ for each group): the control group (group C) and the UC model group (group M). The mice in the UC model group freely drank a 5\% DSS solution for 7 days in order to develop experimental colitis $(1,2,8,9)$. Mice in the control group drank distilled water for the same 7-day span. On day 8 , all mice were sacrificed and colon specimens were collected for research purposes. This study was approved by the Institutional Review Board of the Affiliated Hospital of Nantong University.

Disease activity index (DAI) and histological disease score. The DAI was determined by an investigator blinded to the experiment by scoring the extent of body weight loss, stool hemoccult positivity or gross bleeding and stool consistency, in accordance with the method described by Murano et al (10) (Table I). For histology, the rectum was fixed in $10 \%$ neutral buffered formalin and 4-mm specimens were subjected to hematoxylin and eosin (H\&E) staining. Randomly selected fields $(n=15)$ magnified at $x 100$ were inspected and graded by a pathologist blinded to the treatment instructions (Table II) (5). The mean score in each section was calculated.

Reverse transcription-polymerase chain reaction (RT-PCR). Total RNA from the colonic mucosa was extracted according to standard TRIzol RNA isolation instructions and evaluated with a spectrophotometer for quantity and purity. First strand cDNA was synthesized from $1 \mu \mathrm{g}$ total RNA in a $25-\mu 1$ reaction volume, containing $4 \mu \mathrm{l} 5 \mathrm{X}$ First-Strand Buffer, $0.5 \mu \mathrm{l}$ ribonuclease inhibitor, $2 \mu \mathrm{ldNTP}$ mix, $2 \mu \mathrm{l}$ DTT and $1 \mu \mathrm{l} \mathrm{MMLV}$ Reverse Transcriptase (Sangon Biotech, Shanghai, China). The RT reaction was carried out for $60 \mathrm{~min}$ at $37^{\circ} \mathrm{C}$. PCR products were obtained from $5 \mu \mathrm{l}$ of each cDNA sample in the presence of $2.5 \mu \mathrm{l} 10 \mathrm{X}$ PCR buffer with $\mathrm{MgCl}_{2}, 1 \mu \mathrm{l}$ dNTPs, $2 \mu \mathrm{l}$ sense and antisense primers (each) and $1 \mu 1$ Taq (Sangon Biotech). The housekeeping gene, glyceraldehyde 3-phosphate dehydrogenase (GAPDH), was used as the internal control. The primer sequences for CCL20 were: sense, 5'-AGCAGCAGCAACT ACGACT-3'; and antisense, 5'-TCTTAGGCTGAGGAGG TTCA-3'. The primers for GAPDH were: sense, 5'-ATGGG AAGCTGGTCATCAAC-3'; and antisense, 5'-TTCAGCTCT GGGATGCCT-3'. The sizes of the amplified products were 202 bp for CCL20 and 484 bp for GAPDH.

The amplification was performed under the following conditions: for CCL20, 34 cycles with an annealing temperature of $56^{\circ} \mathrm{C}$ for $30 \mathrm{sec}$; and for GAPDH, 30 cycles with an annealing temperature of $58^{\circ} \mathrm{C}$ for $30 \mathrm{sec}$. Denaturation and extension conditions were $94^{\circ} \mathrm{C}$ for $30 \mathrm{sec}$ and $72^{\circ} \mathrm{C}$ for $40 \mathrm{sec}$, respectively. PCR products were separated by electrophoresis on a $1.5 \%$ agarose gel, stained with ethidium bromide (EB) and
Table I. Disease activity index.

\begin{tabular}{lcll}
\hline Score & $\begin{array}{c}\text { Weight } \\
\text { loss }(\%)\end{array}$ & $\begin{array}{c}\text { Stool } \\
\text { consistency }\end{array}$ & $\begin{array}{c}\text { Occult/gross } \\
\text { bleeding }\end{array}$ \\
\hline 0 & $(-)$ & Normal & Normal \\
1 & $1-5$ & Loose & Guaiac (+) \\
2 & $5-10$ & Diarrhea & Gross bleeding \\
3 & $11-15$ & & \\
4 & $>15$ & & \\
\hline
\end{tabular}

The disease activity index was calculated by: (combined score of

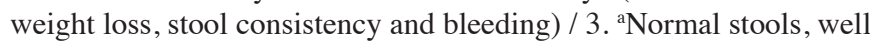
formed pellets; loose, pasty stools which do not stick to the anus; diarrhea, liquid stools that stick to the anus.

Table II. Histological disease score.

\begin{tabular}{ll}
\hline Grade & \multicolumn{1}{c}{ Characteristic } \\
\hline 0 & Normal colonic mucosa \\
1 & Loss of one-third of the crypts \\
2 & Loss of two-thirds of the crypts \\
3 & Lamina propria covered with a single layer of epithe- \\
& lium and mild inflammatory cell infiltration is present \\
4 & $\begin{array}{l}\text { Erosions and marked inflammatory cell infiltration } \\
\text { are present }\end{array}$
\end{tabular}

Randomly selected fields ( $\mathrm{n}=15$; magnification, $\mathrm{x} 100)$ in each section were inspected and graded as described in Patients and methods by a pathologist in our hospital who was blinded to the treatment protocol. By scoring the grades in 15 fields, the mean in each section was calculated.

analyzed with the Gel Doc 2000 system (Bio-Rad, Hercules, CA, USA). The integrated density of the bands was used as a quantitative parameter. CCL20 mRNA levels were expressed as the ratio of band optical intensity to GAPDH. All experiments were performed at least twice and the reported results were reproducible.

Immunohistochemistry. Immunohistochemistry was performed in order to examine the CCL20 protein expression in the colonic mucosa of UC patients and in a mouse model. Briefly, colonic mucosa samples were isolated and immediately fixed in $10 \% \mathrm{pH}$-neutral phosphate-buffered formalin. The fixed tissues were then embedded in paraffin and kept until use. Paraffin sections $(4 \mu \mathrm{m})$ were cut, deparaffinized and hydrated. A Universal Immuno-enzyme Polymer method (Elivison staining) was employed for immunohistochemical staining. Anti-CCL20 polyclonal antibody (R\&D Systems, Minneapolis, MN, USA; dilution, $15 \mu \mathrm{g} / \mathrm{ml}$ ) was used as the primary antibody for 1-h incubation at room temperature. Briefly, staining intensity was scored as 0 (negative), 1 (weak), 2 (medium) or 3 (strong). The extent of staining was scored 
as $0(0 \%), 1(1-25 \%), 2(26-50 \%), 3(51-75 \%)$ or $4(76-100 \%)$ according to the percentage of the positive staining area, in relation to the whole carcinoma area. Subsequently, the sum of the intensity and extent scores was regarded as the final staining score for CCL20. A final score $\geq 3$ was considered positive.

Western blotting. Western blotting was performed on whole cell lysates. Aliquots of total protein (20 $\mu \mathrm{g}$ per lane) were electrophoresed on $10 \%$ SDS-polyacrylamide gradient gels and transferred onto nitrocellulose membranes (Millipore, Billerica, MA, USA). The membranes were incubated for $8 \mathrm{~h}$ at room temperature with anti-CCL20 mAb (R\&D Systems, Alexis Biochemicals, San Diego, CA, USA). Following washing with rinsing buffer, the membranes were incubated with 1:15,000 diluted horseradish peroxidase-conjugated antimouse immunoglobulin antibody (Santa Cruz Biotechnology, Inc., Santa Cruz, CA, USA), followed by development with enhanced chemiluminescence reagents (Amersham Pharmacia Biotech, Little Chalfont, Buckinghamshire, UK).

Statistical analysis. Data are presented as the means \pm standard error (SEM) and analyzed with STATA 7.0 by ANOVA and t-tests between groups. $\mathrm{P}<0.05$ was considered to indicate a statistically significant result.

\section{Results}

DAI. Control group mice had normal diets, activities and bowel movements. Their coats were healthy and overall body quality was slightly increased. Model group mice began to appear anorexic from day 1 and at the same time, their activity decreased, their hair stood vertically, they had abnormal stools and lost weight. By day 3, the mice in the model group began experiencing gross bleeding, occult blood production and more pronounced weight loss. Compared with the control group, the DAI of the model group was significantly higher $(3.48 \pm 0.44$ versus $0.88 \pm 0.22, \mathrm{P}<0.05)$ at day 7 (Table III).

Histological disease score. The colonic mucosal epithelium in the control mice was normal and complete. The inherent
Table III. DAI of DSS group compared with control group $(\mathrm{P}<0.05)$.

\begin{tabular}{lcl}
\hline Day & Control group DAI & DSS group DAI \\
\hline 1 & 1 & 1 \\
2 & 1 & 1 \\
3 & 1.45 & 3.2 \\
4 & 1 & 2.55 \\
5 & 1 & 2.96 \\
6 & 1 & 3.05 \\
7 & 1 & 3.5 \\
\hline
\end{tabular}

DAI, disease activity index; DSS, dextran sulfate sodium.

Table IV. Histological disease score.

\begin{tabular}{lc}
\hline Group $(n=10)$ & Histological disease score $($ mean \pm SD) \\
\hline Control & $0.92 \pm 0.29$ \\
DSS & $3.35 \pm 0.43^{\mathrm{a}}$
\end{tabular}

DSS group compared with control group ${ }^{\mathrm{a}} \mathrm{P}<0.05$. DSS, dextran sulfate sodium.

layered glands were normal and the submucosa revealed only a few inflammatory cells that had infiltrated. However, there was no evidence of erosion or ulcer formation. The colonic mucosa in the model mice was damaged and lost. There was epithelial erosion, ulcer formation and the inherent layered glands were deformed. Additionally, the mucosa was disordered and the submucosa exhibited a high degree of lymphocyte and mononuclear cell infiltration (Fig. 1). The histological scores of colons from mice in the model group were significantly higher than those in the control group $(3.35 \pm 0.43$ versus $0.92 \pm 0.29$, $\mathrm{P}<0.05$; Table IV).
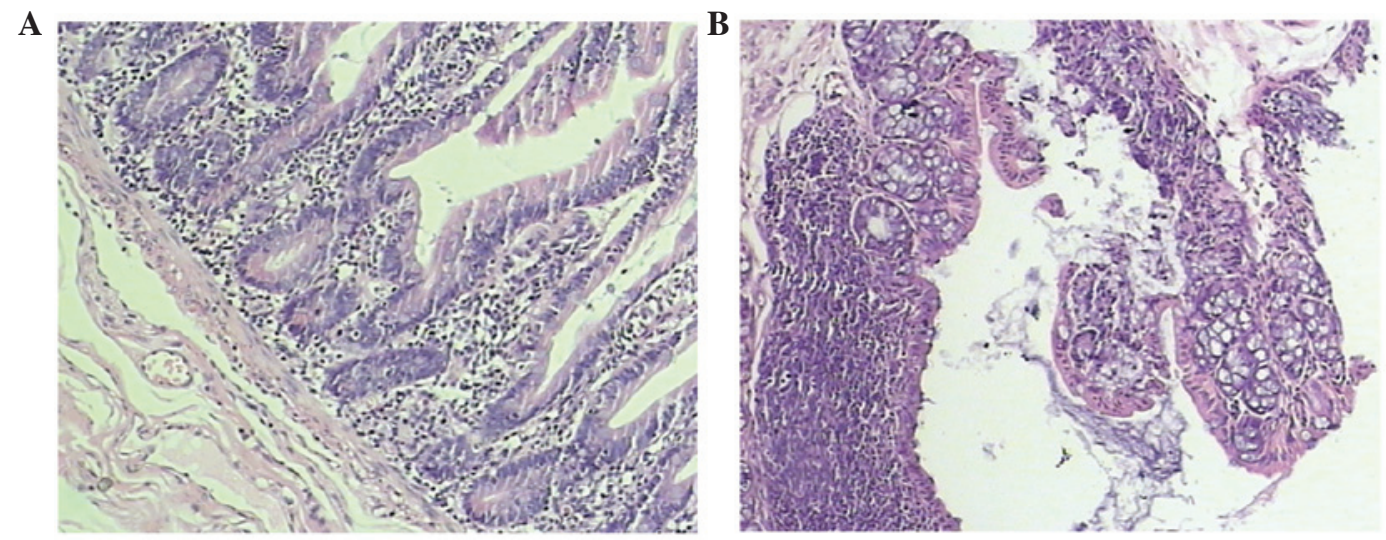

Figure 1. H\&E staining of colonic mucosa in experimental colitis in mice (x400). (A) H\&E staining of control group (original magnification, $\mathrm{x} 400$ ); (B) H\&E staining of colon mucosa of DSS group (original magnification, x400). H\&E, hematoxylin and eosin; DSS, dextran sulfate sodium. 


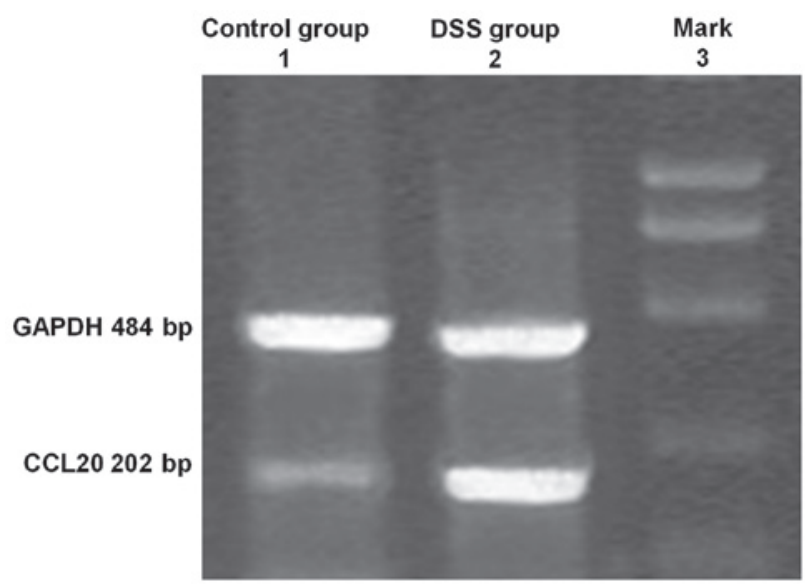

Figure 2. CCL20 mRNA expression detected by RT-PCR. RT-PCR, reverse transcription-polymerase reaction; DSS, dextran sulfate sodium.

$R T-P C R$. CCL20 mRNA was expressed in the colons of all mice in the model and control groups (Fig. 2). However, in the model group, CCL20 mRNA expression was significantly higher than that in the control group and this expression positively correlated with the degree of inflammation $(\mathrm{P}<0.01)$.

Immunohistochemistry in experimental colitis in mice and UC patients. CCL20 was either weakly expressed or not expressed at all in the control mice. However, in the model group mice, CCL20 expression was high (Fig. 3). CCL20 protein expression was exclusively localized to the mucosal epithelium covering the lymphoid follicles.

Immunohistochemical scores of CCL20 in the model group were significantly higher than those in the control group and the scores correlated with inflammation degree $(\mathrm{P}<0.01)$.

In normal colonic mucosa organization, CCL20 was either weakly expressed or not expressed at all. In the bowel mucosa of patients with UC, the CCL20 expression level was 4.52 \pm 1.75 points (Fig. 4B) and this was significantly higher than the levels in the normal control group mice $(0.56 \pm 0.15$ points; Fig. $4 \mathrm{~A})$. This difference was statistically significant $(\mathrm{P}<0.01)$. CCL20 expression in UC increased significantly with the degree of inflammation.

Western blotting. CCL20 was either weakly expressed or not expressed at all in the control group. However, in the model
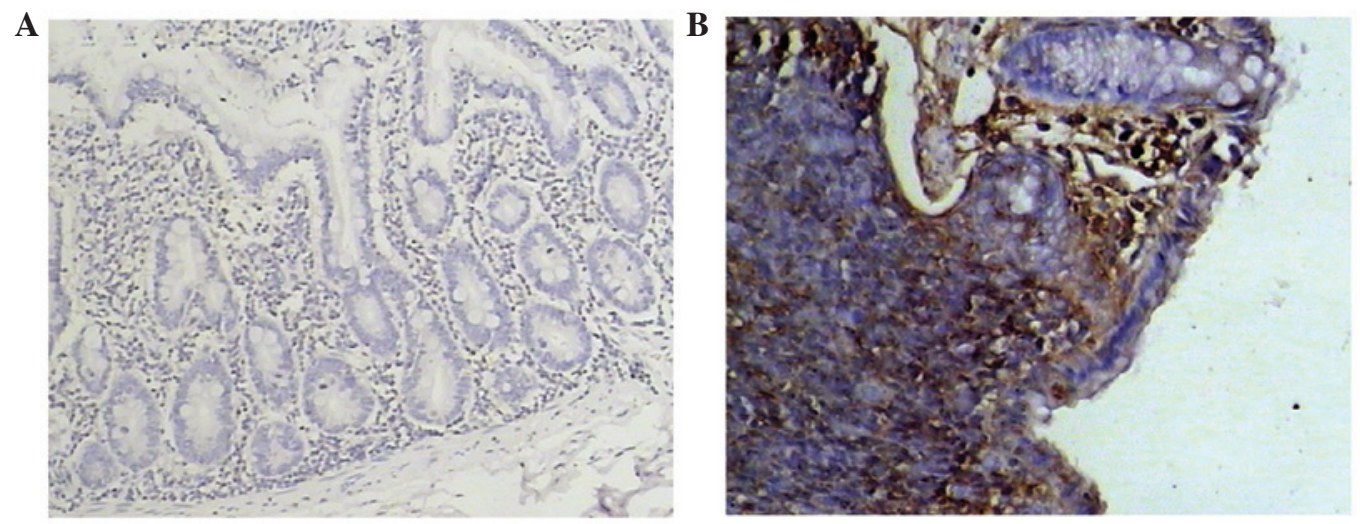

Figure 3. CCL20 protein expression detected by immunohistochemistry in experimental colitis in mice. (A) CCL20 protein in control group; (B) CCL20 protein in DSS group. DSS, dextran sulfate sodium.

A

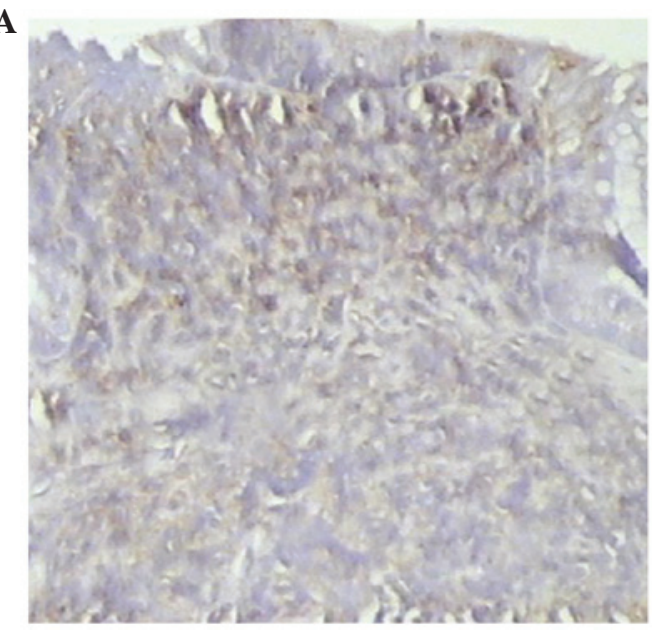

B

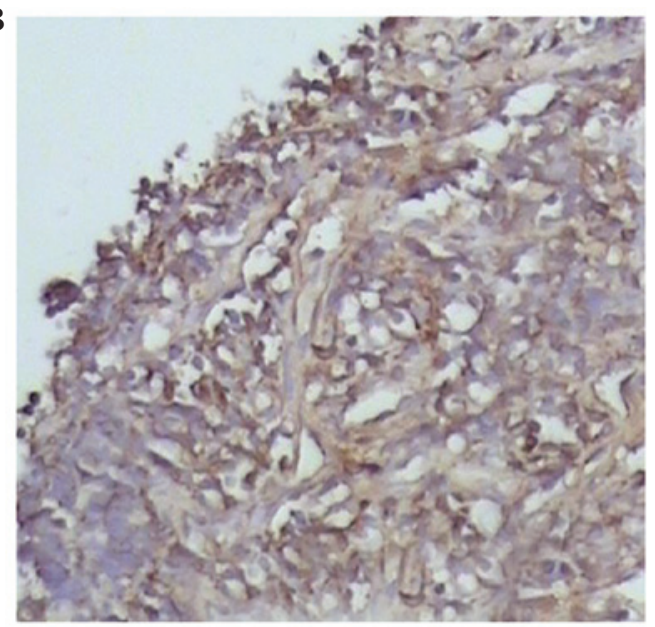

Figure 4. CCL20 protein expression detected by immunohistochemistry in UC patients. (A) CCL20 protein in control patients; (B) CCL20 protein in UC patients. UC, ulcerative colitis. 


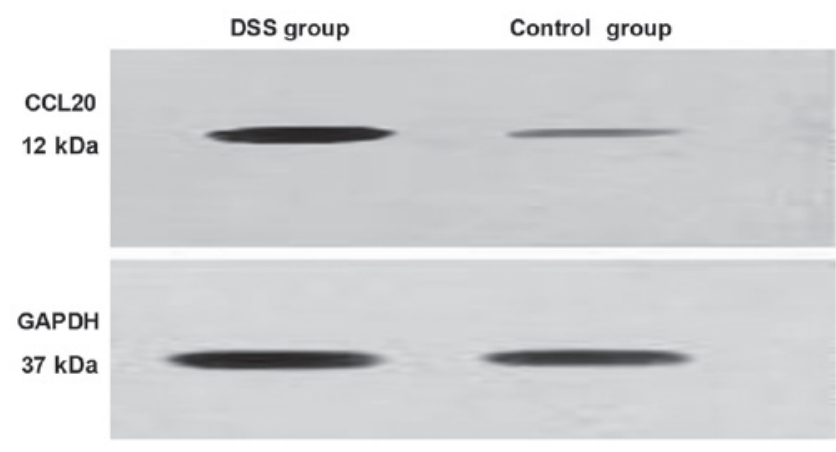

Figure 5. CCL20 protein expression detected by western blotting. DSS, dextran sulfate sodium.

group, CCL20 was expressed at high levels (Fig. 5). That is, CCL20 protein expression in the model group was significantly higher than that in the control group and positively correlated with the degree of inflammation $(\mathrm{P}<0.01)$.

\section{Discussion}

Chemokines are a relatively recently identified family of approximately 40 chemotactic, $7-10-\mathrm{kDa}$ peptides, which have been implicated in the pathophysiology of UC. The 40 chemokines identified in humans are classified into 4 families, designated $\mathrm{CXC}, \mathrm{CC}, \mathrm{C}$ and $\mathrm{CX} 3 \mathrm{C}$, where $\mathrm{X}$ is another amino acid, depending upon the spacing of the $2 \mathrm{~N}$-terminal cysteine residues. Chemokines attract inflammatory cells to a particular location and activate them. Chemokines are produced by a wide variety of cells, including the inflammatory cells present in UC lesions, fibroblasts, endothelial cells and epithelial cells, all of which are abundant in the gastrointestinal system (3-5,10-18).

The main functions of intestinal epithelial cells are absorption and secretion in order to keep the intestinal microenvironment stable. The intestinal immune system is delicately balanced with factors that promote and hinder inflammatory responses. If this balance is disrupted, the extensive, non-specific activation of inflammatory cells results in the production and release of destructive immune molecules and inflammatory factors (19). These factors include activated macrophages and $\mathrm{T}$ lymphocytes, chemotactic factors that promote inflammation and other factors involved in the expression of class II MHC molecules (6). Under normal circumstances, such inflammation is inhibited by interleukin (IL)-4, IL-1 receptor antagonists, IL-10 and transforming growth factor (TGF)- $\beta$ 1. However, in diseased states, these factors cannot fulfill their biological roles (20). In the case of inflammation or infection by pathogenic microorganisms, epithelial cells attract neutrophils, lymphocytes and other inflammatory cells to the bowel mucosa. Additionally, surface cytokines release factors, including IL-1 $\alpha$ and tumor necrosis factor (TNF) $\alpha$. The activation of NF- $\kappa \mathrm{B}$ further raises CCL20 levels and the expression of CCR6 (21). For similar reasons, UC results in significantly increased levels of CCL20. As a result, imDC chemotactic lymphocytes gather in the bowel wall and worsen the inflammation. DCs are antigen cells that have strong antigen processing functions. Dendritic progenitor cells exist in the blood circulation and also settle in the gastrointestinal tract, epithelial tissue of the respiratory tract, urinary and reproductive systems, the heart, liver, kidney and in other essential organs. In the presence of various stimulants, DCs gradually mature through the lymphatic and blood circulation to the lymph nodes (14). Mature DCs (mDCs) activate $\mathrm{T}$ lymphocytes to induce immune responses and lead to the expression of significant stimulatory molecules, including CD80, CD86, CD83, CD54 and CD40 (7,14,22-24). Although the causes may be different, the process of inflammation is uniform, involving the CD4 T cell differentiation of auxiliary T cells (1) and Th1 or Th2. UC is usually thought to occur via gut mucosal inflammation mediated by Th2. It has been previously demonstrated that DC infiltration was positively correlated with the quantity and severity of the inflammatory response (13).

The current study demonstrates that, in the DSS-induced colitis mouse model, CCL20 is overexpressed. By contrast, CCL20 levels were low or undetectable in the control mice. In UC patients, CCL20 was also highly expressed and revealed a positive correlation with the degree of inflammation. The overexpression of CCL20 is a significant factor in the occurrence and development of UC by promoting imDCs in the colonic mucosa by CCR6 and facilitating lymphocytes to lead to the damage of colonic mucosa. Thus, CCL20 reflects the degree and severity of UC disease, suggesting that it is means of monitoring the degree of inflammation. It is possible that altering CCL20 may become an effective treatment method for patients with UC.

In conclusion, CCL20 appears to play a significant role in UC. CCL20 activates DC lymphocytes that then lead directly to the pathological changes of UC. CCL20 reflects the degree of inflammation, which may be used to evaluate disease severity and is a potential therapeutic target.

\section{Acknowledgements}

This study was supported by the Natural Science Foundation of Nantong University (10Z061).

\section{References}

1. Stevceva L, Pavli P, Husband AJ and Doe WF: The inflammatory infiltrate in the acute stage of the dextran sulphate sodium induced colitis: B cell response differs depending on the percentage of DSS used to induce it. BMC Clin Pathol 1: 3, 2001.

2. Bennink RJ, Hamann J, de Bruin K, ten Kate FJ, van Deventer SJ and te Velde AA: Dedicated pinhole SPECT of intestinal neutrophil recruitment in a mouse model of dextran sulfate sodium-induced colitis. J Nucl Med 46: 526-531, 2005.

3. Hyun JG, Lee G, Brown JB, et al: Anti-interferon-inducible chemokine, CXCL10, reduces colitis by impairing T helper-1 induction and recruitment in mice. Inflamm Bowel Dis 11: 799-805, 2005

4. Rivera-Nieves J, Ho J, Bamias G, et al: Antibody blockade of CCL25/CCR9 ameliorates early but not late chronic murine ileitis. Gastroenterology 131: 1518-1529, 2006.

5. Farooq SM, Stillie R, Svensson M, Svanborg C, Strieter RM and Stadnyk AW: Therapeutic effect of blocking CXCR2 on neutrophil recruitment and dextran sodium sulfate-induced colitis. J Pharmacol Exp Ther 329: 123-129, 2009.

6. MacDermott RP: Alterations of the mucosal immune system in inflammatory bowel disease. J Gastroenterol 31: 907-916, 1996.

7. Lee HJ, Choi SC, Lee MH, et al: Increased expression of MIP-3alpha/CCL20 in peripheral blood mononuclear cells from patients with ulcerative colitis and its down-regulation by sulfasalazine and glucocorticoid treatment. Inflamm Bowel Dis 11: $1070-1079,2005$. 
8. ten Hove T, Drillenburg P, Wijnholds J, Te Velde AA and van Deventer SJ: Differential susceptibility of multidrug resistance protein-1 deficient mice to DSS and TNBS-induced colitis. Dig Dis Sci 47: 2056-2063, 2002.

9. te Velde AA, de Kort F, Sterrenburg E, et al: Comparative analysis of colonic gene expression of three experimental colitis models mimicking inflammatory bowel disease. Inflamm Bowel Dis 13: 325-330, 2007.

10. Murano M, Maemura K, Hirata I, et al: Therapeutic effect of intracolonically administered nuclear factor kappa B (p65) antisense oligonucleotide on mouse dextran sulphate sodium (DSS)-induced colitis. Clin Exp Immunol 120: 51-58, 2000.

11. Boirivant M, Fuss IJ, Ferroni L, De Pascale M and Strober W: Oral administration of recombinant cholera toxin subunit B inhibits IL-12-mediated murine experimental (trinitrobenzene sulfonic acid) colitis. J Immunol 166: 3522-3532, 2001.

12. Koga H, Sakisaka S, Ohishi M, et al: Expression of cyclooxygenase-2 in human hepatocellular carcinoma: relevance to tumor dedifferentiation. Hepatology 29: 688-696, 1999.

13. Banks C, Bateman A, Payne R, Johnson P and Sheron N: Chemokine expression in IBD. Mucosal chemokine expression is unselectively increased in both ulcerative colitis and Crohn's disease. J Pathol 199: 28-35, 2003.

14. Watanabe S, Yamakawa M, Hiroaki T, Kawata S and Kimura O: Correlation of dendritic cell infiltration with active crypt inflammation in ulcerative colitis. Clin Immunol 122: 288-297, 2007.

15. Uguccioni M, Gionchetti P, Robbiani DF, et al: Increased expression of IP-10, IL-8, MCP-1, and MCP-3 in ulcerative colitis. Am J Pathol 155: 331-336, 1999.

16. MacDermott RP, Sanderson IR and Reinecker HC: The central role of chemokines (chemotactic cytokines) in the immunopathogenesis of ulcerative colitis and Crohn's disease. Inflamm Bowel Dis 4: 54-67, 1998.
17. Buanne P, Di Carlo E, Caputi L, et al: Crucial pathophysiological role of CXCR2 in experimental ulcerative colitis in mice. J Leukoc Biol 82: 1239-1246, 2007.

18. Zimmerman NP, Vongsa RA, Wendt MK and Dwinell MB: Chemokines and chemokine receptors in mucosal homeostasis at the intestinal epithelial barrier in inflammatory bowel disease. Inflamm Bowel Dis 14: 1000-1011, 2008.

19. Sartor RB: Cytokines in intestinal inflammation: pathophysiological and clinical considerations. Gastroenterology 106: 533-539, 1994.

20. Ashwood P, Harvey R, Verjee T, Wolstencroft R, Thompson RP and Powell JJ: Functional interactions between mucosal IL-1, IL-ra and TGF-beta 1 in ulcerative colitis. Inflamm Res 53: 53-59, 2004.

21. Izadpanah A, Dwinell MB, Eckmann L, Varki NM and Kagnoff MF: Regulated MIP-3alpha/CCL20 production by human intestinal epithelium: mechanism for modulating mucosal immunity. Am J Physiol Gastrointest Liver Physiol 280: G710G719, 2001.

22. Jin Y, Fuller L, Ciancio G, et al: Antigen presentation and immune regulatory capacity of immature and mature-enriched antigen presenting (dendritic) cells derived from human bone marrow. Hum Immunol 65: 93-103, 2004.

23. Kaser A, Ludwiczek O, Holzmann S, et al: Increased expression of CCL20 in human inflammatory bowel disease. J Clin Immunol 24: 74-85, 2004

24. He C, Zhang SL, Hu CJ, Tong DW and Li YZ: Higher levels of CCL20 expression on peripheral blood mononuclear cells of chinese patients with inflammatory bowel disease. Immunol Invest 39: 16-26, 2010 